\title{
Cardiac Markers in scorpion envenomed children in some hospitals
}

in Sohag.

\author{
Mohamed Abd El-Aal ${ }^{(1)}$ Ahmed MonirHegab ${ }^{(2)}$,Ahmed AhmedAllam ${ }^{(3)}$ Sally \\ Shaaban Amin(4), \\ ', Sally Shaaban Amin ${ }^{(4)}$ \\ (1) Professor and head of Pediatric department, Faculty of Medicine, Sohag \\ University, Sohag, Egypt \\ (2) Lecturer, Pediatric Department, Faculty of Medicine, Sohaguniversit, Sohag, \\ Egypt \\ (3)Lecturer of Clinical Pathology, Faculty of Medicine, Sohag University, Egypt \\ (4) Pediatric Department, Faculty of Medicine, Sohag University, Sohag, Egypt
}

\section{Abstract}

Background:Scorpionism is an endemic health problem in Upper Egypt.This study shows theepidemiological,clinical and laboratory manifestations of scorpion envenomation and identify factors that are predictive of severe cases.

Methods: Prospective study had been performed at Emergency Unit in the Sohag university hospital and Sohag General Hospital from January to December , 2016. It included 50 patients with definite history of scorpion sting envenomation.Theepidemiological,clinical,andlaboratory findings of patientswererecorded.

Results: We found that $44 \%$ had abnormal serum troponin and 58\% had abnormal serum CKmb. Also 54\% had abnormal serum lactate dehydrogenase and $44 \%$ had abnormal serum myoglobin. ECG study revealed changes in the severe group in the form of low QRS, ST segment change, sinus tachycardia and ventricular ectopics. Severe group showed significant reduction in the \%SF and LVEF in the comparison to the mild/moderate group.

Conclusions:The present study is demonstrated that $\mathrm{cTnI}$ is a highly specific and sensitive indicator for myocardial injury and adverse outcome in victims of scorpion envenomation. So cTnI may be used in these cases for both diagnosis and prognosis. The use of cTnI in the immediate assessment of patients with severe systemic envenomation appears warranted to identify those at risk of myocardial injury.

\section{Introduction:}

Scorpion envenomation is an important public health hazard in tropical and subtropical regions (1). Envenomation by scorpions can result in a wide range of clinical effects, including, cardiotoxicity, neurotoxicity, respiratory dysfunction and multiorgan dysfunction syndrome (MODS)(2).There are 1500 scorpion species known to exist, only 30 are of medical importanc and majority of them produce similar cardiovascular effects. These effects where studied by critical observations of clinical, neurotransmitters studies, radioisotope studies, echocardiography and haemodynamic patterns (3). Regimen including scorpion antivenom, vasodilators, intensive care management have been used to alleviate the systemic effects of envenoming (4).Scorpion antivenom (SAV) use in children was shown to accelerate recovery times(5) . Having achieved significant reduction in mortality in scorpion sting envenomation, the need of the hour is to reduce morbidity due to scorpion sting envenomation, among which myocardial dysfunction is most important(6). Myocardial damage in children may be clinically not clear in a variety of stressful conditions. However, biochemical markers have not been routinely used in children at risk of myocardial damage due to lack of sufficient specificity (7). As the criterion for myocyte injury is not well established, so the need for a specific serum marker for myocardial injury might be useful to 
SOHAG MEDICALJOURNAL

Vol. 22 No. 2 july 2018

\begin{abstract}
augment the clinical and echocardiographic (Echo) diagnosis of myocarditis. Cardiac troponin I (cTnI) have non-cross reactivity with the skeletal muscle proved that $\mathrm{cTnI}$ is an early marker for viral myocarditis(8). Identification of factors that could potentially predict myocardial dysfunction could be helpful in providing appropriate and timely management, thereby reducing the morbidity due to the condition (9). This study shows the epidemiological,clinical and laboratory manifestations of scorpion envenomation and identify factors that are predictive of severe cases.
\end{abstract}

\section{Pateints and methodS:}

observational prospective study conducted in the period from January to December, 2016.at the Emergency Unit in the Sohag university hospital and Sohag General Hospital.It included 50 patients with definite history of scorpion sting envenomation.All patients were subjected to:Full history taking with focus on: Age of the patient, time of sting, site of sting, color of the scorpion, duration between sting and hospital admission and local and systemic symptoms.Thorough clinical examination with focus on:Cardiovascular examination, Glasgow-coma scale (GCS).Assessment of cardiac function:(1)Cardiac markers:Serum cardiac Troponin I (cTnI) was done by the use of immunochemiluminesence micro particle immunoassey reagents by the use of Architect-1000 (Abbott-DalaceUSA).Serum Creatinephospho Kinase
isoenzyme-MB (CpK-MB), Serum Lactate dehydrogenase (LDH) and Serum Myoglobin $(\mathrm{Mb})$ were done by the use Cobas-C 311, a fully automated chemistry analyzer

(Roche-

USA).(2)Electrocardiography(ECG):stan

dard $12 \quad$ leads.(3) $X$ -

ray.(4)Echocardiography (ECHO).The patientsweregroupedaccordingtoagradings ystem

theseveregradegroupincludedpatientswith severe clinical findings,suchasacutepulmonaryedema,myo carditis,cardiopulmonaryfailure,shock, andc oma;themildmoderategradegroupincluded asymptomaticpatients and patientswithlocalormildsystemic findings

\section{Ethical consideration:}

Approval ofSohag faculty of Medicine Research Ethical Committee was taken. written informed consent was taken from the parents.

\section{Statistical analysis:}

Statistical package for social sciences (IBM-SPSS), version 20 IBM- Chicago, USA .Data expressed as mean, standard deviation (SD) for quantitative data, while number and percentage for qualitative data.Student $t$ test was used to compare the means between two groups for quantitative data. Chi square test was used to compare two groups for qualitative data. $\mathrm{P}$ value < 0.05 was considered as statistically significant.

\section{Result:}

This study was conducted on 50 children presented with scorpion envenomation to the Pediatric Emergency Unit in Sohag University Hospital and Sohag General Hospital from January to December, 2016 .Table (1) shows that the mean age of our study group was 6 years, with standard deviation (SD) 3.7 years, and this reflected in wide range from 2 months to 12 years. $60 \%$ of study group were male. All patients were from rural areasand had definite history of scorpion sting. $62 \%$ had sting at day. $86 \%$ of scorpion were black colour and only $14 \%$ were yellow.The mean of time between sting and admission was 48 minute and range from 18 minute to 2.5 hours.The mean of number of scorpion antivenom was 6.5 , and range from 1 to $20.62 \%$ of stings were in lower extremities, $28 \%$ were in upper extremities, and few were in other sites (back, head, and neck).Table (2) shows the symptoms developed in children included in the study. $52 \%$ of envenomation was mild, and $48 \%$ was severe.The most frequent symptoms were tachycardia, vomiting, cold extremities, lacrimation developed in $76 \%, 68 \%, 68 \%, 52 \%$ of patients respectivity while other symptoms were encephalopathy in $42 \%$, agitation in $42 \%$, tachypnea in $40 \%$, myocarditis in $30 \%$, itching in 
SOHAG MEDICAL JOURNAL

Vol. 22 No. 2 july 2018
Cardiac Markers in scorpion envenomed children

Sally Shaaban Amin ${ }^{(4)}$

28\%,salivation in 26\%, pulmonary edema (manifested by: basal crepitations, hypoxemia and respiratory distress) in $18 \%$, abdominal distension in $12 \%$, parathesia in $10 \%$, bronchospasm in $8 \%$, seizure in $6 \%$,mydriasis in $2 \%$. Investigations of all cases are summarized in Table (3) Blood glucose level was markedly increased in the severe group than mild/moderate group $(\mathrm{P}<0.001)$. Values of troponin ,CPK-MB, LDH and Myoglobin in severe group were significantly higher than mild/moderate group $(\mathrm{P}<0.001)$. Pulmonary edema was observed in $\mathrm{X}$-ray of 8 patients in the severe group $(\mathrm{P}=0.001)$. ECG study revealed changes in the severe group in the the form of low QRS,STsegment change sinus tachycardia and ventricular ectopics $(\mathrm{P}<0.001, \mathrm{P}<0.001, \mathrm{P}<0.001$ and $\mathrm{P}=0.001$, respectively). Severe group showed significant reduction in the \% SF in comparison to the mild/moderate group $(\mathrm{P}<0.001)$. Meanwhile, the Severe group showed significant lower value of LVEF in comparison to the mild/moderate group $(\mathrm{P}=0.001)$.

Table (1):Clinical characteristics of study participants.

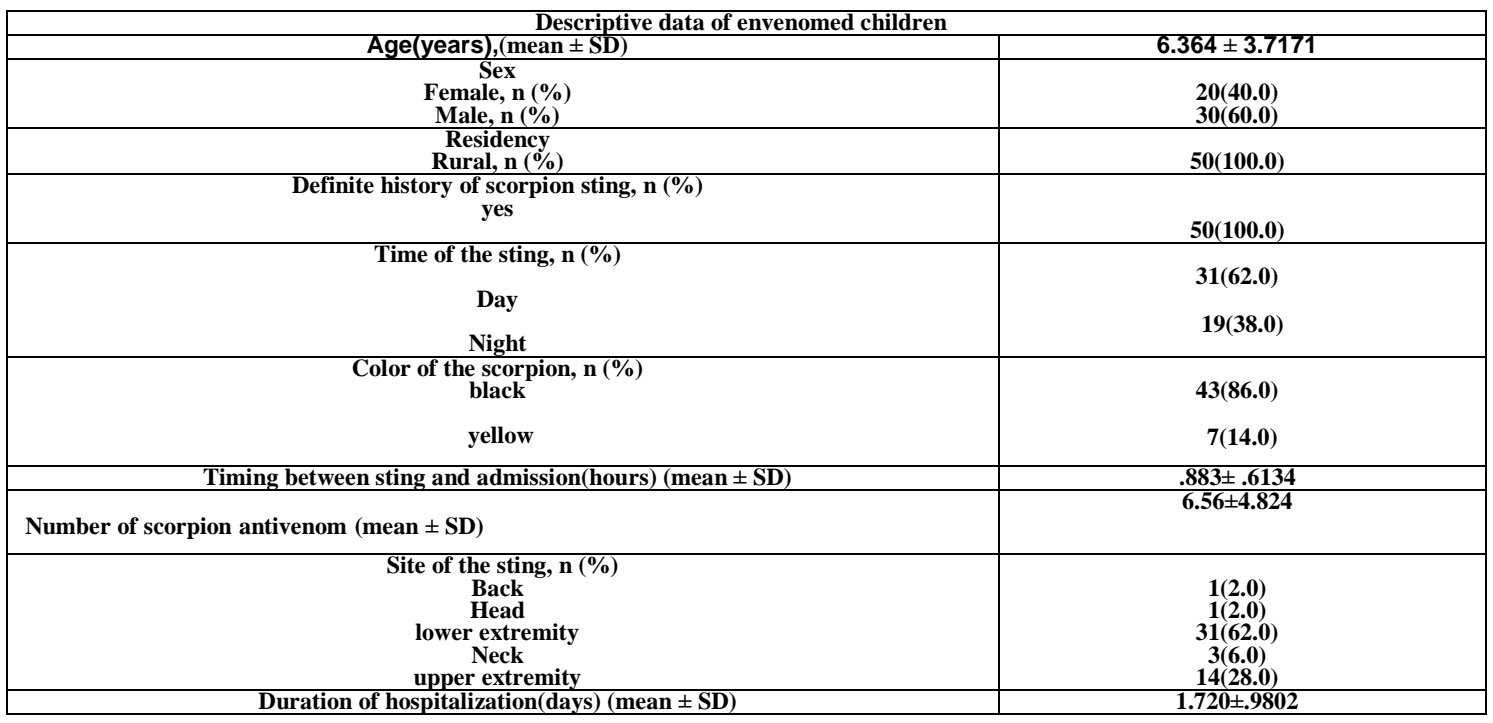

Table (2): Clinical manifestations of scorpion envenomation.

\begin{tabular}{|c|c|}
\hline Clinical manifestations of scorpion envenomed children & n $(\%)$ \\
\hline $\begin{array}{c}\text { Clinical Severity of envenomation } \\
\text { Mild } \\
\text { Severe } \\
\end{array}$ & $\begin{array}{l}26(52.0) \\
24(48.00)\end{array}$ \\
\hline Encephalopathy (disturbed conscious level) & $21(42.0)$ \\
\hline Seizure & 3(6.0) \\
\hline Agitation & 21(42.0) \\
\hline Tachycardia & 38(76.0) \\
\hline Tachypnea & $20(40.0)$ \\
\hline $\begin{array}{c}\text { Pupil (miosis, mydriasis, anisocoria) } \\
\text { (mydriasis) }\end{array}$ & $1(2.0)$ \\
\hline Pulmonary edema & 9(18.0) \\
\hline Myocarditis & 15(30.0) \\
\hline Bronchospasm & $4(8.0)$ \\
\hline Abdominal distension & $6(12.0)$ \\
\hline Vomiting & 34(68.0) \\
\hline Cold extremities & 34(68.0) \\
\hline Salivation & 13(26.0) \\
\hline Lacrimation & $26(52.0)$ \\
\hline Parathesia & $\mathbf{5 ( 1 0 . 0 )}$ \\
\hline Itching & 14(28.0) \\
\hline
\end{tabular}


SOHAG MEDICAL JOURNAL

Vol. 22 No. 2 july 2018
Cardiac Markers in scorpion envenomed children

Sally Shaaban Amin ${ }^{(4)}$

Table (3):Comparison between investigation results in patients with mild to moderate envenomation and those with severe envenomation.

\begin{tabular}{|c|c|c|c|c|}
\hline \multicolumn{2}{|c|}{ Parameter } & Mild-moderate grade (26) & Severe grade (24) & P value \\
\hline \multicolumn{2}{|c|}{ Blood glucose level } & $128.77 \pm 27.26$ & $177.04 \pm 54.99$ & $<0.001$ \\
\hline \multirow[t]{2}{*}{ Serum troponin } & Median & $\begin{array}{c}0.01 \\
(0.001-0.03) \\
\end{array}$ & $0.5(0.01-5)$ & $<0.001$ \\
\hline & Abnormal & $\mathbf{0}$ & $22(91.7 \%)$ & $<0.001$ \\
\hline \multirow[t]{2}{*}{ Creatine kinase } & Median & 22(15-27) & $55(26-270)$ & $<0.001$ \\
\hline & Abnormal & $5(19.2 \%)$ & $24(100 \%)$ & $<0.001$ \\
\hline \multirow[t]{2}{*}{$\begin{array}{l}\text { Lactate dehydro- } \\
\text { genase }\end{array}$} & Median & $546(97-760)$ & $\begin{array}{c}802 \\
(593-1344) \\
\end{array}$ & $<0.001$ \\
\hline & Abnormal & $4(15.4 \%)$ & $23(95.8 \%)$ & $<0.001$ \\
\hline \multirow[t]{2}{*}{ Myoglobin } & Median & $38(19-70)$ & $79.5(26-105)$ & $<0.001$ \\
\hline & Abnormal & $\mathbf{0}$ & $22(91.7 \%)$ & $<0.001$ \\
\hline \multirow[t]{2}{*}{$\mathrm{X}$ ray } & Normal & $26(100 \%)$ & $16(66.7 \%)$ & \multirow[t]{2}{*}{0.001} \\
\hline & Pulmonary edema & $\mathbf{0}$ & $8(33.3 \%)$ & \\
\hline \multirow[t]{5}{*}{ ECG } & Low amp. QRS & $\mathbf{0}$ & $10(41.7 \%)$ & $<0.001$ \\
\hline & ST segment change & $\mathbf{0}$ & $10(41.7 \%)$ & $<0.001$ \\
\hline & Ventricular ectopics & $\mathbf{0}$ & $8(33.3 \%)$ & 0.001 \\
\hline & Sinus tachycard. & $\mathbf{0}$ & $19(79.2 \%)$ & $<0.001$ \\
\hline & $\begin{array}{l}\text { Ventricular } \\
\text { tachycard. }\end{array}$ & $\mathbf{0}$ & $2(8.3 \%)$ & 0.435 \\
\hline \multirow[b]{2}{*}{ ECHO } & LVEF & $71.73 \pm 2.31$ & $65.83 \pm 7.23$ & 0.001 \\
\hline & SF\% & $31.50 \pm 2.35$ & $25.5 \pm 3.48$ & $<0.001$ \\
\hline
\end{tabular}

\section{Discussion:}

Mean of age of our study group was 6 years, with SD 3.7 years, and this reflected in wide range from 2 months to 12 years, $60 \%$ of study group were male, $40 \%$ were female, and all patients were from rural areas, this was with agreement with Chippaux et al. (10) as mean age of the person stung in their study was 48 months, age of the severe group was significantly less than that of the mild- moderate group. The majority of cases $(58.5 \%)$ were boys. Also Mohamad et al. (11) reported in their study that all of the patients lived in rural areas and had a low socioeconomic status. Most of the children typically played outside of their homes.All our patients had definite history of scorpion sting, $62 \%$ had sting at day, $38 \%$ had sting at night, $86 \%$ of scorpion were black colour and only $14 \%$ were yellow.Mean of time between sting and admission was 48 minute and range from 18 minute to 2.5 hours, alsoCesaretli et al. (12) reported that the median duration between sting and hospital admission was longer for the severe group compared with the mildmoderate group, mean of number of scorpion antivenom in our study was 6.5 , and range from 1 to $20.62 \%$ of stings were in lower extremities, $28 \%$ were in upper pulmonary edema, only $30 \%$ of patients had myocarditis, only $8 \%$ had bronchospasm, $68 \%$ of patients had vomiting, $68 \%$ of patients had cold extremities, and few were in other sites (back, head, and neck), this was in agreement with study of Bosnak et al. (13) as in their study the most common sting site involved the extremities, only 2 patients were stung on the neck while sleeping, and 1 of them died. Otero et al. (14) also reported in their results that stings occurred to lower or upper extremities in $86.5 \%$ of the cases. This is likely due to factors including the high level of agricultural activity in rural areas, the frequency of wearing sandals or walking barefoot, the habit of putting on shoes without preshaking them, and hand searching for scorpions in homes (15).In our study $52 \%$ of envenomation was mild, and only $48 \%$ was severe, this was similar to study by Adiguzel et al. (16) as there were 27 patients $(65.9 \%)$ in the mildmoderate group and 14 patients $(34.1 \%)$ in the severe group and majority of stings in both groups $(36.6 \%)$ occurred in July.Regarding clinical manifestations $42 \%$ of our patients had encephalopathy (disturbed conscious level), only 6\% of patients had seizures, $42 \%$ of patients had agitation, $76 \%$ of patients had tachycardia, only $40 \%$ of patients had tachypnea, only $18 \%$ of them had extremities, only $26 \%$ had salivation, only 1 patient of all cases had mydriasis, 52\% of patients had lacrimation, and only $28 \%$ had itching.Our findings confirmed by 
SOHAG MEDICALJOURNAL

Vol. 22 No. 2 july 2018
Isbister et al. , Chippaux et al. $(3,10)$ as the most common findings in their studies was pain, the most common systemic clinical findings werecold extremities, tachypnea, vomiting, sweating, tachycardia, and agitation. All of these systemic findings were increased in the severe group, and the differences from the mild-moderate group were statistically significant. Fever was noted in 7 patients $(17.1 \%)$. In the severe group, 5 patients had fever, and 2 of these patients had mydriasis. One of the patients with mydriasis had a seizure. Both patients with mydriasis died. Myocarditis was frequently observed in patients with fevers, a finding that was statistically significant. The most commonly observed dysrhythmia in all patients was sinus tachycardia, observed in 14 patients (34.1\%) (1).Bosnak et al. (13) reported priapism in $72.4 \%$ patients; in contrast, we observed priapism in $26 \%$ patients. Although fever was observed less frequently in this study compared with other studies $(17,11)$.Petricevich et al. (18) said that in particular, myocarditis was more frequently observed in patients with fever. These results can be explained by the effects of cytokines: proinflammatory cytokines cause fever and other systemic symptoms; and an imbalance between proinflammatory and anti inflammatory mediators may be associated with mortality and morbidity (18).Previous studies on scorpion envenomation reported left ventricular dysfunction, myocarditis, respiratory failure, and pulmonary edema in victims of scorpion stings (19).We found that $56 \%$ had normal serum troponin, and $44 \%$ had abnormal serum troponin with mean $0.486,42 \%$ had normal serum CK, and $58 \%$ had abnormal serum CK with mean 61, SD 70, and range from 15 to 270 . Also $46 \%$ of patients had normal serum lactate dehydrogenase, and $54 \%$ had abnormal serum lactate dehydrogenase with mean 656 , SD 232 , and range from 97 to 1344 , serum myoglobulin was normal in 56\% of patients, and it was abnormal in $44 \%$ of patients with mean 57, SD 25 , and range from 19 to 105.Our findings were in agreement withSofer et al. (20) as they found that leukocyte levels were markedly increased in the severe group, and on admission, their troponin I, CK-MB, and CPK levels were markedly increased. Median troponin I values peaked at 24 hours and then decreased. Two patients had initial troponin levels that were normal.Pulmonary edema was present in only $16 \%$ of our patients which less frequently than reported in other studies and it was observed in all myocarditis cases, however, supporting the hypothesis that pulmonary edema results from cardiac dysfunction (2,21), only $20 \%$ of our patients had ECG low amplitude QRS, only $20 \%$ had ECG changes in ST segment, and only $16 \%$ had ECG ventricular ectopics.In study of Abroug et al. (22) pulmonary edema developed in $9.6 \%$ and dyspnea in $23.0 \%$ oftheir patients. Acute pulmonary edema has been attributed to acute left ventricular failure due to massive release of catecholamines or myocardial injury induced by the venom. Cardiogenic shock and pulmonary edema are the leading causes of death after scorpion envenomation; however, breathing disturbances have also been observed in patients without pulmonary edema or myocardial damage after scorpion envenomation (23).Mean of ECHO left ventricular ejection fraction was 68 , with range from 40 to 78 , however mean of ECHO shortening fraction of left ventricle (SF\%) was 28, and range from 15 to 35 , this was similar to results of Sofer et al. (20) asLVEF was $25 \%$ on echocardiography. Bucaretchi et al. , Izquierdo et al. (24,25)analyzed 1327 patients stung by scorpions in Brazil; found only 1 patient had ventricular tachycardia. 
SOHAG MEDICALJOURNAL

Vol. 22 No. 2 july 2018

\section{Conclusion:}

We found that $44 \%$ had abnormal serum troponin and 58\% had abnormal serum CKmb. Also 54\% had abnormal serum lactate dehydrogenase and $44 \%$ had abnormal serum myoglobin. ECG study revealed changes in the severe group in the form of low QRS, ST segment change, sinus tachycardia and ventricular ectopics. Severe group showed significant reduction in the \%SF and LVEF in the comparison to the mild/moderate group.

\section{Acknowledgments:}

I wish to acknowledge the great help and cooperation offered by the Pediatric Department, Sohag General Hospital.To the head of the department, its staff members, doctors (especially Dr.AymanTahaMoussa) and certainly patients.

\section{References :}

(1)Meki AR, Mohamed ZM, Mohey Eldeen HM. Significance of assessment of serum cardiac troponin I and interleukin- 8 in scorpion envenomed children. Toxicon. 2003;41:129-137.

(2)Bahloul M, Chabchoub I, Chaari A, et al.Scorpion envenomation among children:clinical manifestations and outcome (analysisof685cases). Am JTropMed Hygiene. 2010;83:10841092.

(3)Isbister GK, Bawaskar HS.Scorpion envenomation. $\mathrm{N}$ Engl $\mathrm{J}$ Med. 2014;371:457-63.

(4)Prasad R, Mishra OP, Pandey N, Singh TB. Scorpion sting envenomation in children: Factors affecting the outcome. Indian J Pediatr 2011;78:544-8.

(5)Bawaskar HS, Bawaskar PH. Efficacy and safety of scorpion antivenom plus prazosin compared with prazosin alone for venomous scorpion (Mesobuthustamulus) sting: Randomised open label clinical trial. BMJ. 2011;342:c7136.

(6)Pandi K, Krishnamurthy S, Srinivasaraghavan R, Mahadevan S. Efficacy of scorpion antivenom plus prazosin versus prazosin alone for Mesobuthustamulus scorpion sting envenomation in children: a randomised controlled trial. Arch Dis Child 2014;99:575-80.

(7)Khattabi A oulaymani -Bancheikh R et al. Classification of clinical consequences of scorpion sting: consensus development. Trans RoySoc Trop Med Hyg 2011;105:364-69

(8)Konca C, Tekin M, Turgut M. Doxazosin in the treatment of scorpion envenomation. Indian $\mathbf{J}$ Pediatr 2014;14-1423-6.

(9)Kumar M, Krishnamurthy S, Delhikumar CG, Narayanan P, Biswal N, Srinivasan S. Scrub typhus in children at a tertiary hospital in southern India: clinical profile and complications. J Infect Public Health 2012;5:82-8.

(10)Chippaux

$\mathrm{JP}$, GoyffonM.Epidemiology of scorpion is m:a global appraisal. Acta Tropica. 2008;107:71-79.

(11)Mohamad

IL, ElsayhKI,MohammadHA,etal. Clinical characteristics and outcome of children stung by scorpion.EurJPediatr. 2014;173:815-818.

(12)CesaretliY,OzkanO.Scorpion stings inTurkey: epidemiological and clinical aspects between the years 1995 and 2004. Rev Inst Med Trop Sao Paulo. 2010;52:215-220.

(13)Bosnak M, EceA, Y olbas I, Bosnak V, Kaplan M, Gurkan F. Scorpion sting envenomation in children in southeast Turkey. Wilderness Environ Med. 2009;20:118-124.

(14)Otero R, Navi'o A, Cespedes FA, et al. Scorpion envenoming in two regions of Colombia: clinical, epidemiological and therapeutic aspects. Trans R Soc Trop Med Hyg. 2004;98:742-750.

(15) Ozkan O, Adiguzel S, Yakistiran S, et al. Androctonuscrassicauda (Olivier 1807) scorpionism in the Sanliurfa Provinces of Turkey. ActaParasitolTurcica. 2006;30: 239245.

(16)AdiguzelS,Ozkan O, Inceoglu B. Epidemiological and clinical characteristics of scorpion is $\mathrm{min}$ 
SOHAG MEDICAL JOURNAL

Vol. 22 No. 2 july 2018 children in Sanliurfa, Turkey. Toxicon.

17)Pipelzadeh MH, JalaliA, Taraz M, Pourabbas R, Zaremir- akabadiA.An epidemiological and aclinical study on scorpionism by the Iranian scorpion Hemiscorpiusleptu- rus.Toxicon. 2007;50:984-992.

(18)PetricevichVL.Scorpion venom and the inflammatory response.MediatInflamm. 2010;2010:903295.

(19)Das S, Nalini P, Anantakrishnan S, et al. Cardiac involvement and scorpion envenomation in children. $\mathbf{J}$ Trop Pediatr. 1995;41:338-340.

(20)Sofer S, Zucker N, Bilenko N, et al. The importance of early bedside echocardiography in children with scorpion envenomation.Toxicon. 2013;68:1-8.

(21)Bouaziz M, Bahloul M, Kallel H, et al.Epidemiological, clinical characteristics and outcome of severe scorpion envenomation in South Tunisia:multivariateanalysisof 951 cases. Toxicon. 2008;52:918-926.
2007;49:875-880.

(22)Abroug F, Ayari M, Nouira S, et al. Assessment of left ventricular function in severe scorpion envenomation: combined hemodynamic and echoDoppler study. Intensive Care Med. 1995;3:629-635.

(23)Gueron M, Illia R, Margulia G. Arthropod poisons and the cardiovascular system. Am J Emerg Med. 2000;18:708-714.

(24)Bucaretchi F, Fernandes LC, FernandesCB,etal.Clinical consequences of Tityusbahiensis and Tityusserrulatus scorpion stings in the region of Campinas, south eastern Brazil. Toxicon. 2014;89C:17-25.

(25)Izquierdo LM, RodriguezBuitragoJR.Cardiovascular dysfunction and pulmonary edema secondary to severe envenoming by Tityuspachyurus sting. Casereport.Toxicon. 2012;60:603-606. 